\title{
Inguinal hernia in hemodialysis versus peritoneal dialysis patients: a case-control study
}

\author{
Masataka Banshodani ${ }^{1 *}$, Hideki Kawanishi ${ }^{1,2}$, Misaki Moriishi $^{1}$, Sadanori Shintaku', Shinji Hashimoto ${ }^{1}$ \\ and Masahiro Nishihara ${ }^{3}$
}

\begin{abstract}
Background: Inguinal hernia is common in dialysis patients, especially peritoneal dialysis (PD) patients; however, no previous reports have assessed the incidence, type, treatments, and risk factors of inguinal hernia in hemodialysis (HD) patients. We retrospectively evaluated the differences in these factors between HD and PD patients.

Methods: A total of 2287 HD and 410 PD patients treated between March 2006 and December 2013 were enrolled. The incidence, type (indirect, direct, or combined), and hernioplasty of inguinal hernia as well as demographic characteristics of HD and PD patients were compared.

Results: The incidence of inguinal hernia was lower in HD (0.7 \%) than in PD patients (5.9\%) $(P<0.001)$, and these incidences were higher among men $(P=0.007$ and $P<0.001$, respectively). Of the $16 \mathrm{HD}$ and $24 \mathrm{PD}$ patients with inguinal hernia, 14 and 21 (both $88 \%$ ) underwent hernioplasty. Among them, PD patients demonstrated a higher proportion of indirect hernia (91\%) than HD (47\%) patients $(P=0.003)$. No recurrence was observed in either group. In a multivariate logistic regression model, PD and male sex were risk factors for inguinal hernia (both $P<0.001$ ).

Conclusions: In this study, indirect inguinal hernia was more frequent in PD patients than in HD patients. Male sex and PD are risk factors for inguinal hernia in dialysis patients. Tension-free hernioplasty for inguinal hernia was effective in both HD and PD patients. Our findings can help inform clinicians when selecting the dialysis modality (HD or PD) prior to the initiation of dialysis therapy and guide clinical management practices for inguinal hernias in dialysis patients.
\end{abstract}

Keywords: Hemodialysis, Hernioplasty, Inguinal hernia, Peritoneal dialysis

\section{Background}

Inguinal hernia, which accounts for $75 \%$ of abdominal wall hernias [1], is common in dialysis patients as well as healthy individuals and is caused by abdominal wall fragility [2] and increased intra-abdominal pressure [3]. Aging, male sex, chronic cough [4], prolonged peritoneal dialysis (PD) vintage [5], and polycystic kidney disease [6] have been reported as risk factors of abdominal wall hernia formation. By contrast, being overweight has been reported to reduce the risk of inguinal hernia $[4,7,8]$. However, to the best of our knowledge, no previous reports have focused on the incidence, type (direct, indirect,

\footnotetext{
* Correspondence: m-banshodani@tsuchiya-hp.jp

'Department of Artificial Organs, Akane-Foundation, Tsuchiya General

Hospital, 3-30, Nakajimacho, Naka-ku, Hiroshima 730-8655, Japan

Full list of author information is available at the end of the article
}

or combined), and risk factors of inguinal hernia in hemodialysis (HD) patients.

In this study, we compared the incidence, type, surgical treatments, and risk factors of inguinal hernia between HD and PD patients.

\section{Methods}

\section{Patient identification}

This study was a retrospective analysis of records from Tsuchiya General Hospital collected between March 2006 and December 2013. The management and surgical techniques for dialysis patients with inguinal hernia were established in March 2006. The study was approved by the Tsuchiya General Hospital Institutional Review Board for Human Investigation and performed according to the principles of the Declaration of Helsinki. Written 
informed consent for the procedure was obtained from all patients, and patient anonymity was strictly maintained. We identified HD and PD patients who developed inguinal hernia. Patients with a history of abdominal hernia prior to HD or PD therapy were excluded. Eligible patients were divided into 4 groups, excluding umbilical hernia patients: HD hernia, PD hernia, HD no-hernia, and PD no-hernia. Clinical manifestations were recorded for each patient after a medical chart review. Data were collected at the initiation of HD or PD therapy or the onset of hernia and included demographic characteristics such as age, sex, body mass index (BMI), primary renal disease, history of asthma, and previous abdominal surgery as well as whether hernia was unilateral or bilateral. Data concerning daily peritoneal dialysate volume and the proportion of automated PD (APD) during the study period were also collected.

\section{Methods and techniques for hernioplasty}

A single surgeon with 15 years of experience performed all hernioplasties. Under general anesthesia, a Kugel repair (tension-free hernioplasty) was performed using a Kugel or Polysoft Hernia Patch (polypropylene mesh) (Davol, Cranston, RI, USA) [9]. The type of inguinal hernia (direct, indirect, or combined) was identified at hernioplasty. During hernioplasty, great care was taken not to open the peritoneum to avoid dialysate leakage.

\section{Clinical outcomes}

We reviewed post-procedural complications including wound bleeding, infection, hernia recurrence, and postoperative 90-day survival in HD and PD patients and reviewed peritonitis, dialysate leakage, PD dysfunction, PD withdrawal, and PD vintage after hernioplasty in PD patients.

\section{Statistical analysis}

Data were analyzed using the Statistical Package for the Social Sciences version 14 software (SPSS Inc., Chicago, IL, USA) and expressed as the number of participants and percentage of the study population or as means and ranges. To analyze factors associated with the development of hernias, Student's $t$ test and chi-square test were used for continuous and categorical variables, respectively. Logistic regression analyses were performed to identify independent risk factors for the development of hernia. Values of $P<0.05$ were considered statistically significant.

\section{Results}

\section{Patient characteristics}

At our hospital, $2287 \mathrm{HD}$ patients (1401 men $(P=0.31)$; mean age, 65; range, 18-99 years at the initiation of HD therapy) and 410 PD patients (257 men; mean age, 61; range, $24-98$ years $(P<0.001)$ at the initiation of $\mathrm{PD}$ therapy) were treated during the study period. Patient characteristics are summarized in Table 1 . The primary renal diseases in the HD and PD patients were diabetes mellitus ( $N=937$ and 121, respectively), chronic glomerulonephritis $(N=605$ and 206), nephrosclerosis $(N=204$ and 17), polycystic kidney disease $(N=58$ and 7$)$, immunoglobulin A nephropathy $(N=46$ and 9$)$, others $(N=185$ and 11$)$, and unknown $(N=252$ and 39$)$. The rate of diabetes mellitus diagnoses was significantly higher in the HD group $(P<0.001)$. Among the HD and PD patients, $16(0.7 \%)$ and $24(5.9 \%)$ developed inguinal hernia, respectively $(P<0.001)$.

In these $16 \mathrm{HD}$ and $24 \mathrm{PD}$ hernia patients, the primary renal diseases were diabetes mellitus $(N=4$ and 15 , respectively), chronic glomerulonephritis ( $N=5$ and 7$)$, nephrosclerosis $(N=4$ and 1$)$, polycystic kidney disease ( $N=1$; PD group), renal hypoplasia ( $N=1$; HD group), and unknown ( $N=2$; HD group). At the onset of hernia, the mean ages of the HD and PD patients were 66 (22-91) and $66(42-82)$ years, respectively $(P=0.9)$. Fifteen HD and $21 \mathrm{PD}$ patients had unilateral hernia, and $1 \mathrm{HD}$ and 3 PD patients had bilateral hernias, respectively $(P=0.5)$. The mean duration from initiation of dialysis therapy to onset of hernia in the HD patients (64 (range, 5-148) months) was significantly longer than that in the PD patients $(24(1-101)$ months; $P=0.004)$. There were significantly higher proportions of men in both the HD and PD hernia groups $(100 \%(P<0.001)$ and $88 \%(P=0.007))$ compared to the no-hernia groups (61 and $62 \%$, respectively). The mean BMI of patients at the onset of hernia (23.4 (19.1-33.0) $\mathrm{kg} / \mathrm{m}^{2}$ ) was similar to that during the initiation of PD therapy $\left(23.1(18.4-32.2) \mathrm{kg} / \mathrm{m}^{2}\right.$; $P=0.58$ ), while the mean BMI of patients at the onset

Table 1 Patient characteristics and incidence of inguinal hernia by dialysis status

\begin{tabular}{llll}
\hline Variable & $\begin{array}{l}\mathrm{HD} \\
(N=2287)\end{array}$ & $\begin{array}{l}\text { PD } \\
(N=410)\end{array}$ & P value \\
\hline Age at dialysis initiation, & 65 & 61 & $<0.001$ \\
years (range) & $(18-99)$ & $(24-98)$ & \\
Male, N (\%) & $1401(61)$ & $257(63)$ & 0.31 \\
Primary renal disease & & & \\
Diabetes mellitus, N (\%) & 937 & 121 & 0.001 \\
& $(41)$ & $(29.5)$ & \\
Chronic glomerulonephritis, N (\%) & $605(26)$ & $206(50)$ & \\
Nephrosclerosis, N (\%) & $204(9)$ & $17(4)$ & \\
Polycystic kidney disease, N (\%) & $58(3)$ & $7(2)$ & \\
IgA nephropathy, N (\%) & $46(2)$ & $9(2)$ & \\
Others, N (\%) & $185(8)$ & $11(3)$ & \\
Unknown, N (\%) & $252(11)$ & $39(9.5)$ & \\
Patients with incident inguinal hernia, & $16(0.7)$ & $24(5.9)$ & $<0.001$ \\
$N$ (\%) & & & \\
\hline
\end{tabular}

$H D$ hemodialysis, $\lg A$ immunoglobulin $A, P D$ peritoneal dialysis 
of hernia (19.9 (18.3-21.4) $\left.\mathrm{kg} / \mathrm{m}^{2}\right)$ was significantly lower than that during the initiation of HD therapy (20.7 (19.6-22.6) $\left.\mathrm{kg} / \mathrm{m}^{2} ; P<0.001\right)$. The mean BMI of the HD hernia group was lower than that of the PD hernia group at both dialysis initiation $(P=0.03)$ and hernia onset $(P=0.004)$ (Table 2$)$.

The rates of asthma in both the HD and PD hernia groups $(13 \%(N=2 ; P=0.9)$ and $13 \%(N=3 ; P=0.5))$ were similar to those in the no-hernia groups $(11 \%$ $(N=252)$ and $19 \%(N=71)$, respectively). The proportion of previous surgery in the PD hernia group (30\% $(N=7))$ was similar to that in the PD no-hernia group (25\% $(N=93 ; P=0.46))$, while the proportion of previous abdominal surgery in the HD hernia group (31\% $(N=5))$ was significantly higher than that in the HD no-hernia group $(12 \%(N=265 ; P=0.04))$.

In the PD patients, the mean daily dialysate volume was 5.3 (range, 1.0-15.5) L/day; $16 \%$ of patients underwent APD. The mean daily dialysate volumes in the PD hernia and PD no-hernia groups were 4.8 (range, 3.0-7.5) and $5.4(1.0-15.5) \mathrm{L} /$ day, respectively $(P=0.12)$. The rates of APD before the onset of hernias in the PD hernia and PD no-hernia groups were 9 and $17 \%$, respectively $(P=0.25)$.

In logistic regression analyses, $\mathrm{PD}$ (odds ratio (OR), 9.8; $95 \%$ confidence interval $(\mathrm{CI}), 5.1-19.4 ; P<0.001)$ and male sex (OR, 7.8; $95 \% \mathrm{CI}, 2.8-32.7 ; P<0.001)$ were independent risk factors for the development of inguinal hernia. The other variables were not determinants (Table 3).

\section{Hernioplasty}

Hernioplasty data are summarized in Table 4. Of the 16 HD and 24 PD patients with inguinal hernia, 14 and 21

Table 2 Patient characteristics with incident inguinal hernia by dialysis status

\begin{tabular}{|c|c|c|c|}
\hline Variable & $\begin{array}{l}H D \\
(N=16)\end{array}$ & $\begin{array}{l}P D \\
(N=24)\end{array}$ & $P$ value \\
\hline Male, $N(\%)$ & $16(100)$ & $21(88)$ & 0.07 \\
\hline$P$ value vs. no-hernia male (\%) & $<0.001(61)$ & $0.007(62)$ & \\
\hline Age at hernia onset, years (range) & $\begin{array}{l}66 \\
(22-91)\end{array}$ & $\begin{array}{l}66 \\
(42-82)\end{array}$ & 0.9 \\
\hline Unilateral/bilateral hernia, $N$ & $15 / 1$ & $21 / 3$ & 0.5 \\
\hline $\begin{array}{l}\text { Duration from dialysis initiation to } \\
\text { hernia onset, months (range) }\end{array}$ & $\begin{array}{l}64 \\
(5-148)\end{array}$ & $\begin{array}{l}24 \\
(1-101)\end{array}$ & 0.004 \\
\hline \multicolumn{4}{|l|}{ Body mass index, $\mathrm{kg} / \mathrm{m}^{2}$ (range) } \\
\hline Dialysis initiation & $\begin{array}{l}20.7 \\
(19.6-22.6)\end{array}$ & $\begin{array}{l}23.1 \\
(18.4-32.2)\end{array}$ & 0.03 \\
\hline Hernia onset & $\begin{array}{l}19.9 \\
(18.3-21.4)\end{array}$ & $\begin{array}{l}23.4 \\
(19.1-33.0)\end{array}$ & 0.004 \\
\hline $\begin{array}{l}P \text { value (dialysis initiation } \\
\text { vs. hernia onset) }\end{array}$ & $<0.001$ & 0.58 & \\
\hline
\end{tabular}

$H D$ hemodialysis, $P D$ peritoneal dialysis, vs. versus
Table 3 Risk factors for inguinal hernia development in dialysis patients

\begin{tabular}{lcll}
\hline Variable & \multicolumn{3}{l}{ Inguinal hernia } \\
\cline { 2 - 4 } & Odds ratio & $95 \% \mathrm{Cl}$ & $P$ value \\
\hline PD & 9.8 & $5.1-19.4$ & $<0.001$ \\
Age at dialysis initiation & 0.6 & $0.1-3.8$ & 0.6 \\
Male & 7.8 & $2.8-32.7$ & $<0.001$ \\
Diabetes mellitus & 0.8 & $0.4-1.6$ & 0.5 \\
Asthma & 0.8 & $0.3-2.0$ & 0.7 \\
Previous abdominal surgery & 0.8 & $0.3-1.9$ & 0.6 \\
\hline
\end{tabular}

$C /$ confidence interval, $P D$ peritoneal dialysis

(both $88 \%$ ) underwent a Kugel repair. The total of 22 hernias in the 21 PD patients included 20 (91\%) indirect, 1 direct, and 1 combined hernia, while the total of 15 hernias in the 14 HD patients included 7 (47\%) indirect, 6 direct, and 2 combined hernias. The incidence of indirect hernias was significantly higher in the PD group $(P=0.003)$. The median duration from the onset of inguinal hernia to hernioplasty in the $\mathrm{HD}$ and $\mathrm{PD}$ patients was 30 (range, 0.7-261) and $8(0.2-75)$ months, respectively.

\section{Clinical outcomes}

No recurrence was observed in either the HD or PD groups. In 2 patients, PD therapy was discontinued due to complications within 90 days of hernioplasty: 1 case of peritonitis and 1 of lower abdominal discomfort. In 1 patient, PD therapy was withdrawn due to peritonitis prior to hernioplasty. The mean PD vintage after hernioplasty was 28 (range, 1-62) months. The overall postoperative 90 -day survival rate was $100 \%$ in both groups (Table 4).

Table 4 Hernioplasty for dialysis patients with inguinal hernia and clinical outcomes

\begin{tabular}{|c|c|c|c|}
\hline Variable & $\begin{array}{l}\mathrm{HD} \\
(N=16)\end{array}$ & $\begin{array}{l}P D \\
(N=24)\end{array}$ & $P$ value \\
\hline Patients with hernioplasty, N (\%) & $14(88)$ & $21(88)$ & \\
\hline Number of inguinal hernia, $N$ & 15 & 22 & \\
\hline \multicolumn{4}{|l|}{ Hernia type, $N(\%)$} \\
\hline Indirect & $7(47)$ & $20(91)$ & 0.003 \\
\hline Direct & 6 & 1 & \\
\hline Combined & 2 & 1 & \\
\hline $\begin{array}{l}\text { Duration from hernia onset to } \\
\text { hernioplasty, months (range) }\end{array}$ & $\begin{array}{l}30 \\
(0.7-261)\end{array}$ & $\begin{array}{l}8 \\
(0.2-75)\end{array}$ & 0.2 \\
\hline Recurrence, $N$ & 0 & 0 & \\
\hline 90-day survival rate, \% & 100 & 100 & \\
\hline
\end{tabular}

$H D$ hemodialysis, $P D$ peritoneal dialysis 


\section{Discussion}

PD patients tend to have a higher incidence of inguinal hernias compared with healthy individuals. The reported incidence of abdominal wall hernias ranges from 2.5 to $25 \%$ in PD patients $[10,11]$. Therefore, the incidence of inguinal hernia in PD patients in our study was consistent with previous reports. By contrast, no previous reports have investigated the incidence of inguinal hernia in HD patients. Moreover, to the best of our knowledge, no previous studies have investigated the incidence of inguinal hernia in healthy Japanese adults, although the hernia is common in healthy individuals. On the other hand, a previous study reported that the incidences of groin hernias in Nepalese (South Asian) men and women were 1.5 and $0.6 \%$, respectively [12]. In our study, the incidence in HD patients $(0.7 \%)$ is similar to that in healthy Western adults and Nepalese, in whom the reported incidence is 1-2 \% [12-14].

Inguinal hernias are classified anatomically as indirect or direct. In the analysis of the Swedish Hernia Register, indirect inguinal hernia accounted for $54 \%$ of groin hernias repaired in men [15]. In our study, there was a higher proportion of indirect hernia in the PD (91\%) than in the HD (47\%) patients treated with hernioplasty. Indirect inguinal hernias in adults are almost always congenital. A shutter mechanism, which is postulated to close the internal inguinal ring to a slit, may be dysfunctional in patients with a patent processus vaginalis $[16,17]$. Our findings suggest that indirect inguinal hernia may not be clinically apparent due to the presence of a small internal ring (i.e., slit) before the initiation of PD therapy and may become clinically detectable as a result of internal ring expansion due to the increase in intra-abdominal pressure from peritoneal dialysate. On the other hand, there is no slit within Hesselbach's triangle, where a direct hernia can occur. Therefore, direct inguinal hernia may be susceptible to intra-abdominal pressure from peritoneal dialysate.

In a previous report, low body weight was associated with abdominal hernia formation in PD patients [18]. In another report, BMI was not a risk factor for hernia development in PD patients [6]. Among adults in the USA, overweight and obesity were associated with a lower incidence of inguinal hernia [4]. No previous reports have focused on risk factors of inguinal hernia in HD patients. In our study, the BMIs of HD patients were lower than those of PD patients at both dialysis initiation and hernia onset, and decreased BMI (i.e., body weight) may be a risk factor for inguinal hernia formation only in HD patients. Our findings in HD patients are similar to those previously reported in healthy individuals [4]. In HD patients, lean or malnourished patients, who have less abdominal muscle and loose connective tissues around the inguinal region, may be associated with the development of inguinal hernia. On the other hand, in PD patients, factors associated with PD may counteract other factors including BMI for the development of inguinal hernia.

Strong associations of abdominal hernias with aging, male sex, chronic cough [4], polycystic kidney disease [19], and PD vintage [5] have been reported. In our study, PD and male sex were risk factors for the development of inguinal hernia. By contrast, aging, asthma, polycystic kidney disease, and previous abdominal surgery were not independent risk factors for the development of inguinal hernia. The reasons for these findings may include aging of the patients undergoing dialysis or the small sample size of patients with PD in our study.

Tension-free hernioplasty with mesh is preferred for the treatment of inguinal hernia because of its association with low hernia recurrence rates $[20,21]$ and has also been recommended for PD patients [22]. In our study, tension-free hernioplasty was performed in both HD and PD patients with inguinal hernia, and no recurrence was observed. Our findings suggest that tensionfree hernioplasty is an effective treatment for inguinal hernia in both HD and PD patients. Although the meshes used for hernioplasty were not associated with peritonitis $[23,24]$, great care should be taken not to open the peritoneum during hernioplasty to avoid peritonitis, since the meshes are a foreign body.

This study has several limitations. First, it was based on a group of patients treated at a single center. Second, the sample size was small. However, our study is valuable in that no previous reports have assessed the incidences, type, surgical treatment, and risk factors of inguinal hernias in both HD and PD patients.

\section{Conclusions}

Indirect inguinal hernia was more frequent in PD patients compared with HD patients. Male sex and PD were risk factors of inguinal hernia in dialysis patients. Tension-free hernioplasty for inguinal hernia was effective in both HD and PD patients. Our findings can help inform clinicians when selecting the dialysis modality (HD or PD) prior to the initiation of dialysis therapy and guide clinical management practices for inguinal hernias in dialysis patients.

\section{Competing interests \\ The authors declare that they have no competing interests.}

\section{Authors' contributions}

MB designed the study, performed the retrospective analysis and interpretation of the data obtained from our institution, and wrote the article. HK designed the study and performed the interpretation of the data. MM, SS, SH, and MN provided the intellectual content of the critical importance to the work described. All authors read and approved the final manuscript.

\section{Acknowledgements}

The authors are very grateful to the dialysis staff who provided data in Tsuchiya General Hospital. 


\section{Author details}

'Department of Artificial Organs, Akane-Foundation, Tsuchiya General Hospital, 3-30, Nakajimacho, Naka-ku, Hiroshima 730-8655, Japan. ${ }^{2}$ Faculty of Medicine, Hiroshima University, 1-2-3 Kasumi, Minami-ku, Hiroshima 734-8551, Japan. ${ }^{3}$ Department of Surgery, Akane-Foundation, Tsuchiya General Hospital, 3-30, Nakajimacho, Naka-ku, Hiroshima 730-8655, Japan.

Received: 25 December 2015 Accepted: 22 March 2016

\section{Published online: 15 June 2016}

\section{References}

1. Kingsnorth A, LeBlanc K. Hernias: inguinal and incisional. Lancet. 2003;362: 1561-71.

2. Colin JF, Elliot P, Ellis H. The effect of uraemia upon wound healing: an experimental study. Br J Surg. 1979;66:793-7.

3. Hendry PO, Paterson-Brown S, de Beaux A. Work related aspects of inguinal hernia: a literature review. Surgeon. 2008;6:361-5.

4. Ruhl CE, Everhart JE. Risk factors for inguinal hernia among adults in the US population. Am J Epidemiol. 2007;165:1154-61.

5. Yang SF, Liu CJ, Yang WC, Chang CF, Yang CY, Li SY, et al. The risk factors and the impact of hernia development on technique survival in peritoneal dialysis patients: a population-based cohort study. Perit Dial Int. 2015:35:351-9.

6. Del Peso G, Bajo MA, Costero O, Hevia C, Gil F, Diaz C, et al. Risk factors for abdominal wall complications in peritoneal dialysis patients. Perit Dial Int. 2003;23:249-54

7. Abramson JH, Gofin J, Hopp C, Makler A, Epstein LM. The epidemiology of inguinal hernia. A survey in western Jerusalem. J Epidemiol Community Health. 1978;32:59-67.

8. Liem MS, van der Graaf Y, Zwart RC, Geurts I, van Vroonhoven TJ. Risk factors for inguinal hernia in women: a case-control study. The Coala Trial Group. Am J Epidemiol. 1997;146:721-6.

9. Kugel RD. Minimally invasive, nonlaparoscopic, preperitoneal, and sutureless, inquinal herniorrhaphy. Am J Surg. 1999:178:298-302.

10. Nelson H, Lindner M, Schuman ES, Gross GF, Hayes JF. Abdominal wall hernias as a complication of peritoneal dialysis. Surg Gynecol Obstet. 1983: 157:541-4.

11. O'Connor JP, Rigby RJ, Hardie IR, Wall DR, Strong RW, Woodruff PW, et al. Abdominal hernias complicating continuous ambulatory peritoneal dialysis. Am J Nephrol. 1986;6:271-4.

12. Stewart BT, Pathak J, Gupta S, Shrestha S, Groen RS, Nwomeh BC, et al. An estimate of hernia prevalence in Nepal from a countrywide community survey. Int J Surg. 2015;13:111-4.

13. Holzheimer RG, Schreiber A. Inguinal hernia and concomitant varicocele mimicking mesh complication. Eur J Med Res. 2003:8:254-6.

14. Jenkins JT, O'Dwyer PJ. Inguinal hernias. BMJ. 2008;336:269-72.

15. Koch A, Edwards A, Haapaniemi S, Nordin P, Kald A. Prospective evaluation of 6895 groin hernia repairs in women. Br J Surg. 2005;92:1553-8.

16. Dubbs W. MacLeod WA, O'Connell TX. Restoration of the shutter mechanism in inguinal herniorrhaphy. Am J Surg. 1980;139:461-2.

17. Read RC. British contributions to modern herniology of the groin. Hernia. 2005;9:6-11

18. Twardowski Z, Khanna R, Nolph KD, Scalamogna A, Metzler MH, Schneider TW, et al. Intraabdominal pressures during natural activities in patients treated with continuous ambulatory peritoneal dialysis. Nephron. 1986;44:129-35.

19. Balda S, Power A, Papalois V, Brown E. Impact of hernias on peritoneal dialysis technique survival and residual renal function. Perit Dial Int. 2013;33:629-34

20. Collaboration EUHT. Repair of groin hernia with synthetic mesh: meta-analysis of randomized controlled trials. Ann Surg. 2002;235:322-32.

21. Bisgaard T, Bay-Nielsen M, Christensen IJ, Kehlet H. Risk of recurrence 5 years or more after primary Lichtenstein mesh and sutured inguinal hernia repair. Br J Surg. 2007;94:1038-40

22. Lewis DM, Bingham C, Beaman M, Nicholls AJ, Riad HN. Polypropylene mesh hernia repair - an alternative permitting rapid return to peritoneal dialysis. Nephrol Dial Transplant. 1998;13:2488-9.

23. Afthentopoulos IE, Panduranga Rao S, Mathews R, Oreopoulos DG. Hernia development in CAPD patients and the effect of 2.5 I dialysate volume in selected patients. Clin Nephrol. 1998:49:251-7.

24. Garcia-Urena MA, Rodriguez CR, Vega Ruiz V, Carnero Hernandez FJ, Fernandez-Ruiz E, Vazquez Gallego JM, et al. Prevalence and management of hernias in peritoneal dialysis patients. Perit Dial Int. 2006;26:198-202.

\section{Submit your next manuscript to BioMed Central and we will help you at every step:}

- We accept pre-submission inquiries

- Our selector tool helps you to find the most relevant journal

- We provide round the clock customer support

- Convenient online submission

- Thorough peer review

- Inclusion in PubMed and all major indexing services

- Maximum visibility for your research

Submit your manuscript at www.biomedcentral.com/submit
Biomed Central 